Submitted to

46th AIAA/ASME/ASCE/AHS/ASC Structures, Structural Dynamics, and Materials Conference

18-21 April 2004

Structures Sessions

\title{
STRUCTURAL VERIFICATION OF THE REDESIGNED SPACE SHUTTLE BIPOD FOAM CLOSEOUT
}

\author{
Eric L. Poole \\ Strength Analysis Group/ED22 \\ NASA Marshall Space Flight Center \\ Marshall Space Flight Center, AL 35812 \\ 256-544-2723 voice \\ 256-544-7234 fax \\ Eric.L.Poole@nasa.gov \\ Patrick R. Rogers \\ 256-544-4632 \\ Pat.Rogers@nasa.gov
}

\begin{abstract}
This document outlines the structural verification approach for the Space Shuttle External Tank Forward Bipod Foam Closeout. Due to the Space Shuttle Columbia accident, debris has become a major concern. The intent of the structural verification is to ensure that any debris shed from the bipod is within acceptable limits. Since cohesive failure due to internal defects was identified as the most likely cause of the STS-107 bipod ramp foam failure, verification for this failure mode receives particular emphasis. However, all failure modes for TPS are considered and appropriate verification rationale is developed for each failure mode.

ET TPS verification is accomplished by a combination of analysis, test, and similarity.

Figure 1 depicts the structural verification of a production design where analysis and test are the primary methods of verification. It can be seen that the successful completion of structural verification is dependent on three main areas:

1. Production process control and quality assurance must ensure that test articles and/or analytical models are representative of (or conservatively envelope) production hardware in terms of geometry, materials and processing. Variability and defects must be considered.

2. Flight environments must be sufficiently characterized to bound driving environments for all failure modes. Applied environments, either test or analytical, must be representative of flight environments and have a load factor that satisfies design requirements.

3. Structural verification must include all failure modes. A comprehensive list of failure modes and the underlying failure mechanisms has been generated based on flight and test experience. Verification tests and / or analyses must address each failure mode.
\end{abstract}


First, the strength of the TPS bondline must be established. The structural capability of the bondline is evaluated when subjected to all operation loads (static, thermal, and dynamic), from manufacturing through re-entry. This evaluation protects against structural failures that lead to large-scale loss of foam and therefore compromise the TPS design requirements of thermal protection and debris generation. The TPS structure must demonstrate a 1.4 Factor of Safety or greater. This factor was chosen based on the guidelines of NASA-STD-5001, which recommends a design and qualification test factor of 1.4 for prototype composite structures. Worst-case environments will be applied for all structural evaluations. Minimum material capability will be used in analysis of the structure. Test demonstrated factors of safety will be assessed for the effects of material variability, either using a knockdown factor or accepting some additional risk. If an initial evaluation of the TPS structure does not demonstrate adequate strength margin, the problem must be addressed. Actions taken to solve the problem can either be an improvement to the flight hardware through redesign or process improvements, or a refinement of the Factor of Safety evaluation through improved testing or analysis.

The failure modes of bondline delamination, bondline adhesion, and cohesion will be addressed in the Factor of Safety calculations. However, cohesive failure cannot be predicted in foam above $200 \mathrm{~F}$, because the behavior of the foam in this temperature range is not understood well enough. Therefore, testing will be used to determine the debris generated by this failure mode and the resulting test data will be used for verification that the TPS meets its requirements.

Once the TPS has demonstrated the 1.4 Factor of Safety on bondline capability and possibly cracking, the limit on allowable defects must then be determined. First, the flaw tolerance of the TPS structure is determined either by analysis or by testing hardware with representative defects. In either case, the tolerance must be determined using minimum material properties and worst-case environments including the effects of cyclic loading. The flaw tolerance will be a function of location on the tank and depth inside the TPS. The allowable flaw size can then be derived from the debris requirements.

The size and shape of defects in the flight hardware are determined by process demonstration. Non-Destructive Evaluation (NDE) of the flight hardware would be the preferred method of determining defect size, but currently no technology exists which can find defects with confidence. Demonstration is achieved by fabricating a flight configuration TPS structure using a certified process, and then performing dissection to find any manufactured defects. Several structures will be dissected to provide a data set for statistical evaluation. If an upper bound defect size can be established using statistical analysis, then the upper bound defect size can be compared directly to the flaw allowable size of the structure. If the allowable flaw size is larger than upper bound defect size, then the structure is certified for flight. However, if the statistical analysis of the manufactured defect sizes is inconclusive, a 2.0 factor will be applied to the maximum defect size discovered during dissections. This factor guards against the possibility of having a larger defect than any found during process demonstration. In this case, if the allowable flaw is at least two times greater than the manufactured defect size, then the TPS is certified for flight.

"Divoting" is a term used to describe a cohesive failure modes that results in a piece of debris roughly the size and shape of a grass divot in the game of golf, or larger. The failure starts from an existing flaw in the foam. The flaw has internal pressure, which can build through aeroheating of the foam or because of the boiling of cryopumped/cryoingested air. The drop in ambient pressure during ascent creates a differential pressure load on the foam. Once the 
pressure differential is sufficient, the foam above the void fractures and debris is expelled. Figure 4 shows a divot produced in laboratory testing.

For the evaluation of divoting, TPS defect tolerance will be determined by critical flaw testing and/or fracture mechanics analysis. Defect size in the flight hardware will be verified by demonstration and process control or measured by NDE, if available. At this time, the maximum possible defect size has not been determined. Therefore, the safety factor on allowable void size has been increased to 2.0. This factor is applied to the largest void found during process demonstration. When a statistical upper found for internal defects is determined, the safety factor will be lowered to 1.0 .

\section{FIGURES}

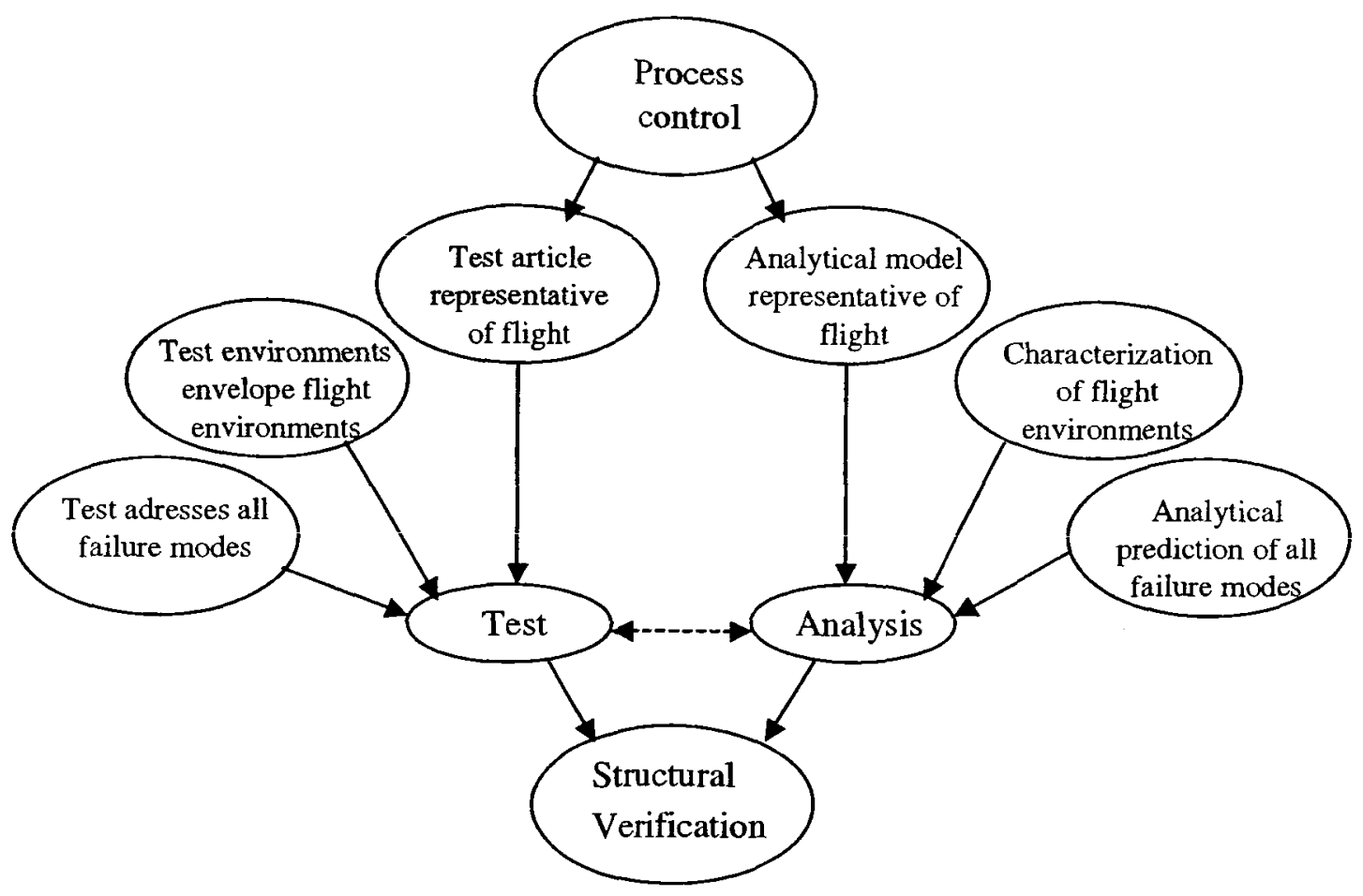

Figure 1. Structural Verification Process 


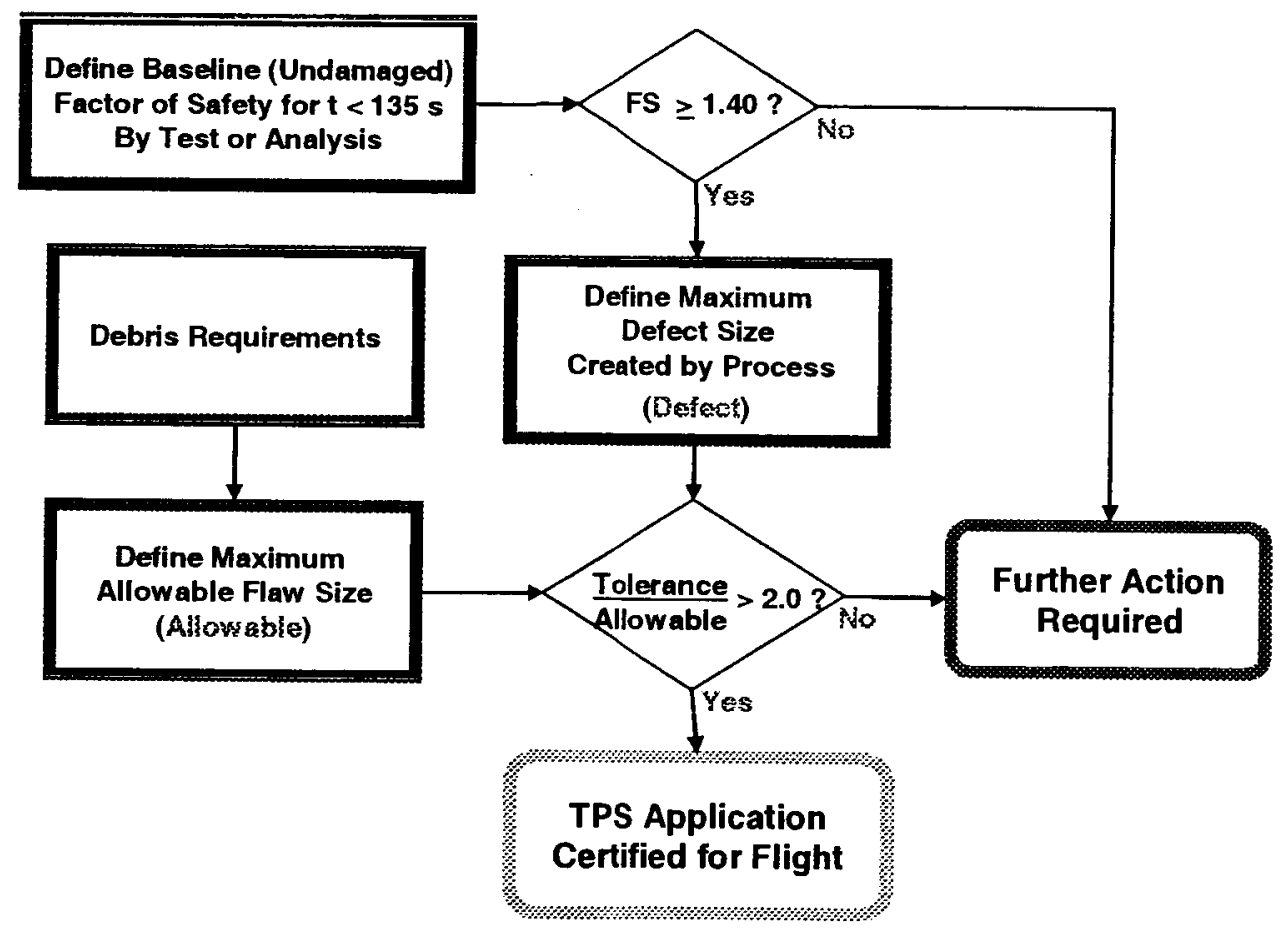

Figure 2. Structural Verification Logic 


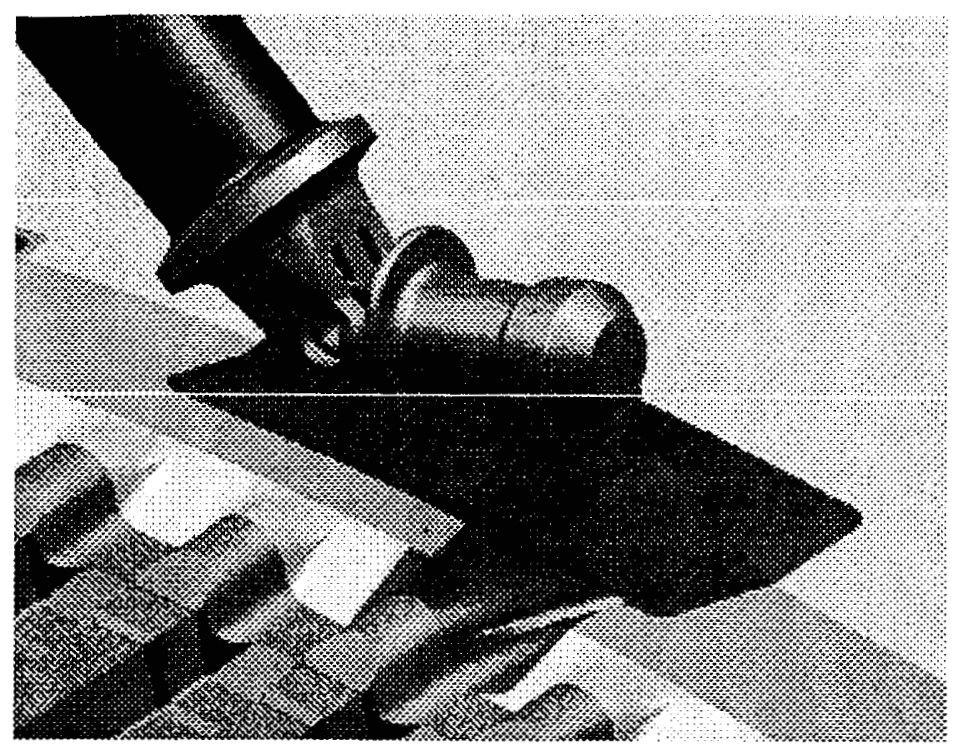

Figure 3. Redesigned Forward Bipod Closeout

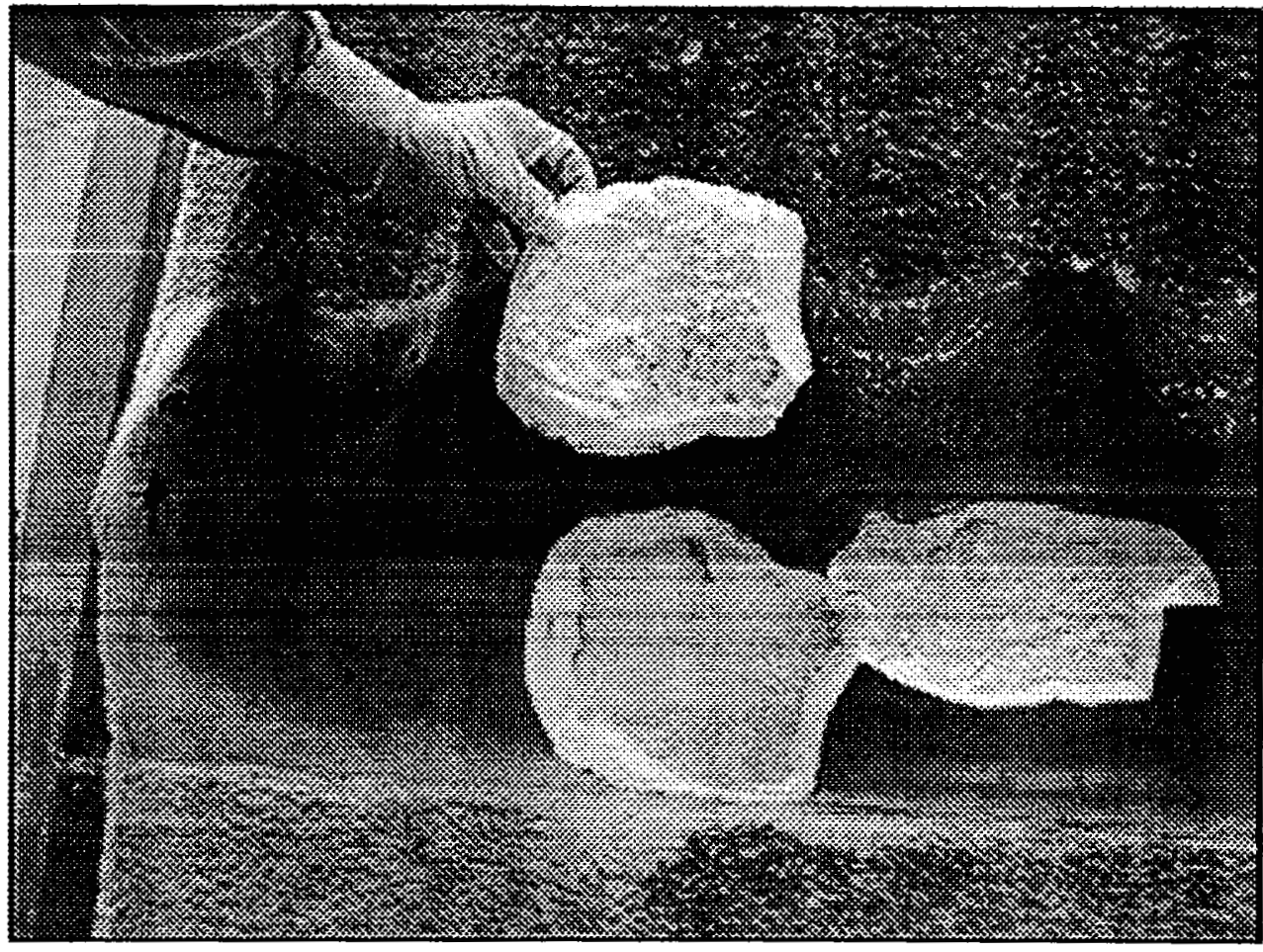

Figure 4: Divot produced during Intertank/LH2 Flange Enhancement program 